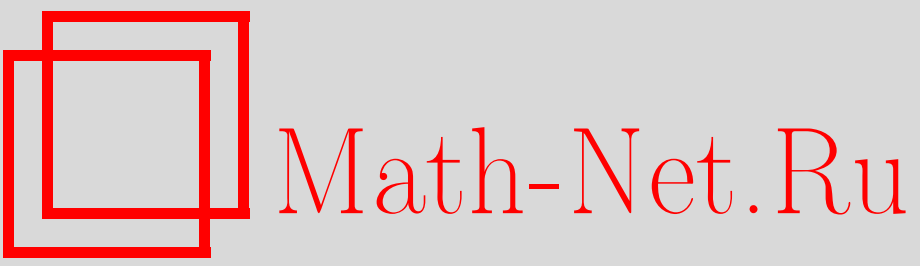

К. А. Свешников, П. К. Силаев, Квазиточное решение релятивистского конечноразностного аналога уравнения Шредингера для прямоугольной потенциальной ямы, ТМФ, 2002, том 132, номер 3, 408-433

DOI: https://doi.org/10.4213/tmf371

Использование Общероссийского математического портала Math-Net.Ru подразумевает, что вы прочитали и согласны с пользовательским соглашением

http://www. mathnet.ru/rus/agreement

Параметры загрузки:

IP : 107.22 .136 .117

26 апреля 2023 г., 12:14:55 
ТЕОРЕТИЧЕСКАЯ

И МАТЕМАТИЧЕСКАЯ

ФИЗИКА

Том 132, № 3

сентябрь, 2002

(C) 2002 г.

К.А. Свешников* , П.К. Силаев*

\section{КВАЗИТОЧНОЕ РЕШЕНИЕ РЕЛЯТИВИСТСКОГО КОНЕЧНО-РАЗНОСТНОГО АНАЛОГА УРАВНЕНИЯ ШРЕДИНГЕРА ДЛЯ ПРЯМОУГОЛЬНОЙ ПОТЕНЦИАЛЬНОЙ ЯМЫ}

Рассмотрена корректная постановка спектральной задачи для релятивистского аналога одномерного уравнения Шредингера, содержащего вместо дифференциальных операторы конечного чисто мнимого сдвига $\exp ( \pm i \hbar d / d x)$. Для потенциалов типа прямоугольной ямы найдены эффективные методы решения такой задачи, позволяющие находить спектр и исследовать свойства волновых функций в широком диапазоне изменения параметров. Показано, что свойства решений уравнений такого типа существенно зависят от соотношения между $\hbar$ и параметрами потенциала, причем вполне возможна ситуация, когда при $\hbar \ll 1$ решение тем не менее будет принципиально отличаться от своего шредингеровского аналога.

Ключевые слова: релятивистская задача о связанных состояниях, квантование полей в лоренцевых базисах, уравнения в конечных разностях с мнимым шагом.

\section{1. ВВЕДЕНИЕ}

Как известно, при реализации квазипотенциального подхода Логунова-Тавхелидзе к описанию релятивистской двухчастичной системы [1] в рамках гамильтоновой формулировки [2], когда все импульсы лежат на массовом гиперболоиде, после разложения по главной серии унитарных неприводимых представлений группы Лоренца (так называемым лоренцевым базисам) в эффективном конфигурационном пространстве возникает аналог нерелятивистского уравнения Шредингера, в котором радиальная часть свободного гамильтониана представляет из себя комбинацию операторов конечного сдвига $\exp ( \pm i d / d r)[3]-[5]$. Разложение по лоренцевым базисам, приводящее к уравнениям движения для инвариантных переменных, в которых дифференциальные операторы по радиальной переменной заменяются на аналогичные операторы конечных чисто мнимых сдвигов аргумента, возникает также и в задаче ковариантного описания квантованного поля в окрестности классической частицеподобной (т.е. протяженной, но локализованной) составляющей [6]. В обоих случаях за счет таких чисто мнимых конечных сдвигов аргумента динамические уравнения оказываются по сушеству функциональными, и

\footnotetext{
* Московский государственный университет, Москва, Россия. E-mail: costa@bog.msu.su
} 
по целому ряду принципиальных аспектов радикально отличаются от своих дифференциальных аналогов. В частности, как для корректной постановки, так и для решения таких уравнений необходимо находить оригинальные приемы и привлекать как аналитические, так и численные методы, не являющиеся стандартными для задач квантовой теории. Это обусловлено прежде всего тем обстоятельством, что до настоящего времени общих способов решения не разработано даже для линейных уравнений такого типа, что вообще является характерной чертой уравнений в конечных разностях [7]. Как следствие свойства решений таких уравнений могут значительно отличаться от их дифференциальных аналогов, что приводит к существенным изменениям их физической интерпретации.

Настоящая работа посвящена исследованию наиболее простого по форме, но тем не менее содержащего самые специфические особенности уравнений такого типа, релятивистского конечно-разностного аналога одномерного уравнения Шредингера, который имеет вид

$$
\frac{1}{\hbar^{2}}[\psi(x-i \hbar)+\psi(x+i \hbar)-2 \psi(x)]+V(x) \psi(x)=E \psi(x),
$$

где $\hbar$ имеет смысл комптоновской длины $\lambda_{\mathrm{C}}$ приведенной массы соответствуюшей двухчастичной задачи в рамках квазипотенциального подхода на массовой поверхности [3]-[5] или массы классической составляющей при ковариантном квантовании поля в ее окрестности [6]. Мы, однако, будем исходить из более формальной постановки задачи и рассматривать $\hbar$ просто как параметр мнимого сдвига аргумента волновой функции, считая последний безразмерным. Тем самым безразмерными будут как $\hbar$, так и все остальные параметры, используемые в работе.

Для корректной формулировки уравнения (1.1) необходимо с самого начала оговорить два взаимосвязанных вопроса. Первый из них состоит в том, в каком смысле следует понимать выражение $\psi(x \pm i \hbar)$. Здесь мы будем исходить из анализа, проведенного в работах [8] в рамках задачи о ковариантном квантовании поля в окрестности $c$-числовой составляющей, и рассматривать только такие функции $\psi(x)$, которые имеют аналитическое продолжение на полосу $|\operatorname{Im} z|<\hbar$. Тогда следует считать, что

$$
\psi(x \pm i \hbar)=\lim _{\varepsilon \rightarrow+0} \psi[x \pm i(\hbar-\varepsilon)] .
$$

Кроме того, мы введем дополнительное условие, что при $|z| \rightarrow \infty$ в пределах этой полосы $\psi(z) \rightarrow 0$, а на действительной оси функции $\psi(x)$ должны быть квадратично-интегрируемы, как и волновые функции дискретного спектра в квантовой механике.

Вторая проблема состоит в том, что всякое решение уравнения (1.1) определено лишь с точностью до $i \hbar$-периодического множителя. Условие аналитичности $\psi(z)$ в полосе $|\operatorname{Im} z|<\hbar$ эту неоднозначность практически ликвидирует, так как целые $i \hbar$-периодические функции в окрестности бесконечно удаленной точки имеют как минимум экспоненциально растушую асимптотику, в то время как мероморфные - полюсы в полосе периодов. Поэтому умножение на такие функции запрешено либо асимптотикой, либо условием аналитичности $\psi(z)$ в полосе $|\operatorname{Im} z|<\hbar$. Исключение составляет, по-видимому, достаточно экзотический случай, когда функция $\psi(z)$, будучи аналитической в полосе 
$|\operatorname{Im} z|<\hbar$, убывает при $|z| \rightarrow \infty$ в пределах полосы быстрее экспоненты (например, га-

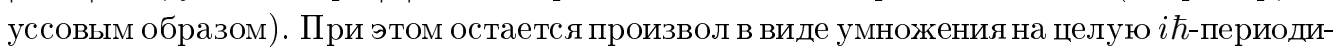
ческую функцию типа $e^{2 \pi n z / \hbar}$, где $n$ - целое число. Однако, по крайней мере, для тех потенциалов $V(x)$, которые будут рассматриваться в настоящей работе, такая ситуация не реализуется, и условие аналитичности в полосе $|\operatorname{Im} z|<\hbar$ оказывается достаточным для выделения единственного решения уравнения (1.1) с требуемыми асимптотическими свойствами.

Заметим, что условию аналитичности $\psi(z)$ в полосе $|\operatorname{Im} z|<\hbar$ можно придать достаточно ясный физический смысл [8]. Для этого введем в рассмотрение функционал

$$
K[\psi]=-\frac{1}{\hbar^{2}} \int d x[\psi(x+i \hbar)-\psi(x)]^{*}[\psi(x-i \hbar)-\psi(x)]
$$

и потребуем, чтобы при переходе в импульсное представление он имел вид

$$
K[\widetilde{\psi}]=\frac{2}{\hbar^{2}} \int d k(\operatorname{ch} \hbar k-1)|\widetilde{\psi}(k)|^{2},
$$

где $\widetilde{\psi}(k)$ - фурье-образ функции $\psi(x)$, и был при этом конечен на допустимых $\psi(x)$. Тогда функционал $K[\psi]$ автоматически действителен, неотрицателен и по существу представляет из себя однозначно определенный конечно-разностный аналог шредингеровского функционала кинетической энергии, соответствующий уравнению (1.1). Подчеркнем, что просто условия конечности функционала (1.4) недостаточно, так как из него следует аналитичность $\psi(z)$ только в полосе $|\operatorname{Im} z|<\hbar / 2$, что запрешает умножение на мероморфные $i \hbar$-периодические функции, но не обеспечивает аналитичности в полосе $|\operatorname{Im} z|<\hbar$, без которой $\psi(x \pm i \hbar)$ остаются неопределенными символами. Переход же от $(1.3)$ к (1.4) эквивалентен утверждению, что преобразование Фурье для $\psi(x \pm i \hbar)$ дает $e^{ \pm \hbar k} \widetilde{\psi}(k)$, что фактически и есть условие аналитичности в полосе $|\operatorname{Im} z|<\hbar$.

Необходимо также отметить, что совокупность перечисленных условий, накладываемых на функцию $\psi(x)$, сохраняет для уравнения (1.1) ряд наиболее общих свойств его дифференциального аналога, т.е. нерелятивистского одномерного уравнения Шредингеpa

$$
-\psi^{\prime \prime}+V(x) \psi=E \psi,
$$

которое является формальным пределом уравнения (1.1) при $\hbar \rightarrow 0$. Физически этот предел соответствует $\lambda_{\mathrm{C}} \rightarrow 0$, т.е. нерелятивистскому пределу двухчастичной задачи или бесконечно большой массе классической составляюшей поля. Обсуждение вопроса о фактическом статусе такого предельного перехода является одним из предметов настоящей работы.

В частности, для уравнения (1.1) сохраняется ограничение снизу на значения возможных уровней энергии $E$, совпадающее с аналогичным ограничением для уравнения (1.5). Для этого умножим уравнение $(1.1)$ на $\psi^{*}(x)$ и проинтегрируем по $d x$, в результате чего получим

$$
K[\psi]+\int d x V(x)|\psi(x)|^{2}=E \int d x|\psi(x)|^{2}
$$


Поскольку в силу (1.4) кинетическая энергия $K[\psi]$ для допустимых $\psi(x)$ является неотрицательной, то из (1.1a) следует, что $E>V_{\min }$. Заметим, что для нормированных функций $\psi(x)$ выражение (1.1a) является конечно-разностным аналогом энергетического функционала, из которого, как и в нерелятивистской квантовой механике, уравнение (1.1) может быть получено с помошью соответствующего вариационного принципа. Аналогичным образом также можно показать эрмитовость разностного оператора в (1.1), откуда, в частности, следует взаимная ортогональность собственных функций уравнения (1.1), соответствующих различным собственным значениям. Подчеркнем еще раз, что для вывода этих свойств уравнения (1.1) сушественны все сформулированные выше условия на $\psi(x)$.

В то же время между уравнениями (1.1) и (1.5) сушествует и ряд принципиальных отличий. В качестве наглядной демонстрации разницы между ними рассмотрим вопрос о спектре связанных состояний с $E<0$ для $\delta$-образного потенциала

$$
V(x)=-\Omega \delta(x), \quad \Omega>0 .
$$

Как известно, уравнение (1.5) в этом случае имеет один дискретный уровень с энергией

$$
E=-\left(\frac{\Omega}{2}\right)^{2}
$$

а соответствующая волновая функция имеет вид

$$
\psi(x)=A e^{-\Omega|x| / 2} .
$$

При этом выражения (1.7) и (1.8) имеют место для всех значений величины "потенциала" $\Omega>0$, причем с ростом последнего волновая функция становится все более локализованной в окрестности начала координат, а сам уровень сдвигается вниз по закону (1.7).

В отличие от уравнения (1.5), для уравнения (1.1) уже в этом простейшем случае решение сушественно зависит от соотношения между параметрами $\hbar$ и $\Omega$, а точнее от соотношения между $\hbar \Omega$ и $2 \pi$. Сначала расмотрим случай

$$
\hbar \Omega \leqslant 2 \pi .
$$

Решение уравнения (1.1) как в этом, так и в остальных случаях, которые будут рассматриваться в дальнейшем, удобно начать с нахождения функции Грина для оператора $\Lambda$

$$
(\Lambda \psi)(x)=\frac{1}{\hbar^{2}}[\psi(x-i \hbar)+\psi(x+i \hbar)-2 \psi(x)]-E \psi(x) .
$$

Для рассматриваемого случая (1.9) имеем

$$
G(x)=\frac{\hbar}{2 \operatorname{sh} \hbar \varkappa} \frac{\operatorname{sh}[(\pi / \hbar-\varkappa) x]}{\operatorname{sh}[(\pi / \hbar) x]},
$$

где

$$
|E|=\frac{2}{\hbar^{2}}(1-\cos \hbar \varkappa)=\frac{4}{\hbar^{2}} \sin ^{2}\left(\frac{\hbar \varkappa}{2}\right), \quad 0<\hbar \varkappa \leqslant \pi .
$$


При этом $|E|$ ограничено сверху величиной $4 / \hbar^{2}$, что вполне естественно, поскольку ограничена величина $\Omega$.

С помошью функции Грина оператора (1.10) для уравнения (1.1) с потенциалом (1.6) находим, что соответствующая волновая функция будет пропорциональна $G(x)$, именно

$$
\psi(x)=A \frac{\operatorname{sh}[(\pi / \hbar-\varkappa) x]}{\operatorname{sh}[(\pi / \hbar) x]}
$$

а уровень энергии $E$ определяется из трансцендентного уравнения

$$
\sin \hbar \varkappa=\frac{\hbar \Omega}{2 \pi}(\pi-\hbar \varkappa)
$$

Из (1.13) очевидно, что найденная таким образом функция $\psi(x)$ удовлетворяет всем предьявляемым к решению уравнения (1.1) требованиям и единственна, так как умножение даже на целую $i \hbar$-периодическую функцию $e^{2 \pi x / \hbar}$ минимальной степени роста запрешено асимптотическим условием для $\psi(x)$. При $|x| \gg \hbar / \pi$ волновая функция (1.13) превращается в убывающую экспоненту $A e^{-\varkappa|x|}$ и отличается от шредингеровской волновой функции (1.8) только скоростью убывания, однако в области $|x| \leqslant \hbar / \pi$ функция (1.13), будучи аналитической, ведет себя бесконечно гладким образом, а функция (1.8) имеет разрыв производной при $x=0$. Аналогичное утверждение относится и к поведению функций Грина уравнений (1.1) и (1.5). Следует отметить, что наличие такой переходной области размером $O(\hbar / \pi)$ в окрестности скачка потенциала является общей характерной чертой решений уравнения (1.1), обусловленной условием аналитичности решений. Появление этой области можно интерпретировать и как результат “сглаживания" сингулярностей потенциала за счет квантовых флуктуаций, приводящих к невозможности его точной локализации на расстояниях порядка эффективной комптоновской длины волны системы, что еще раз подчеркивает существенно релятивистскую природу уравнения (1.1).

Далее легко видеть, что на интервале $0<\hbar \varkappa \leqslant \pi$ уравнение $(1.14)$ может иметь только один корень, причем условие (1.9) есть условие его сушествования. При этом на всем интервале $0<\hbar \varkappa \leqslant \pi$ зависимость величин $\varkappa$ и $E$ от $\Omega$ является по-прежнему монотонной, но становится существенно более нелинейной по сравнению с (1.7), а при $ћ \Omega \rightarrow 2 \pi$ они достигают своих максимальных для этой области значений $\varkappa \rightarrow \pi / \hbar$ и $E \rightarrow 4 / \hbar^{2}$, соответственно. Поэтому с ростом $\Omega$ волновая функция (1.13), как и шредингеровская функция (1.8), становится все более локализованной, но теперь уже в пределах области размером $O(\hbar / \pi)$, а не в окрестности точки $x=0$.

Рассмотрим теперь вопрос о взаимосвязи между решениями уравнений (1.1) и (1.5) для этого случая. Легко видеть, что переход от (1.12)-(1.14) к (1.7), (1.8) реализуется при выполнении одновременно двух условий: $\hbar \rightarrow 0$ и $\hbar \Omega \rightarrow 0$. Иными словами, уравнение (1.5) представляет собой нерелятивистский предел уравнения (1.1) при $\hbar \rightarrow 0$ только при наличии определенных ограничений сверху на величину $\Omega$. Более того, на уровне волновых функций этот предел является несобственным, так как происходит смена степени гладкости функций с бесконечной до разрыва в первой производной.

Теперь рассмотрим ситуацию, когда

$$
\hbar \Omega>2 \pi \text {. }
$$


В этом случае уравнение (1.1) по-прежнему имеет для каждого значения $\Omega$ единственное решение с требуемыми свойствами, но при этом уровни энергии лежат в области $|E|>$ $4 / \hbar^{2}$. Положим поэтому

$$
|E|=\frac{2}{\hbar^{2}}(1+\operatorname{ch} \hbar \alpha)=\frac{4}{\hbar^{2}} \operatorname{ch}^{2}\left(\frac{\hbar \alpha}{2}\right), \quad \alpha>0,
$$

тогда функция Грина оператора (1.10) для этого случая имеет вид

$$
G(x)=\frac{\hbar}{2 \operatorname{sh} \hbar \alpha} \frac{\sin \alpha x}{\operatorname{sh}[(\pi / \hbar) x]} .
$$

Единственное решение уравнения (1.1), удовлетворяюшее сформулированным выше требованиям, снова оказывается пропорциональным $G(x)$ :

$$
\psi(x)=A \frac{\sin \alpha x}{\operatorname{sh}[(\pi / \hbar) x]},
$$

а значения уровней энергии находятся из трансцендентного уравнения

$$
\operatorname{sh} \hbar \alpha=\frac{\hbar \Omega}{2 \pi} \hbar \alpha
$$

которое для каждого $\hbar \Omega$ имеет только одно решение, причем условие (1.15) является условием его разрешимости. Заметим, что выражения (1.16)-(1.19) суть аналитические продолжения соотношений (1.11)-(1.14) на комплексные значения параметра $\varkappa$, именно

$$
\varkappa \rightarrow \frac{\pi}{\hbar}+i \alpha
$$

Из (1.16)-(1.19) очевидно, что в этой области значений $\hbar \Omega$ свойства волновой функции и поведение уровня энергии существенно отличаются от предыдущего случая. В частности, вместо монотонного убывания с ростом $|x|$, которое имело место для волновой функции (1.13), функция (1.18) осциллирует с периодом $2 \pi / \alpha$ и амплитудой, которая убывает как $e^{-(\pi / \hbar)|x|}$, и от величины "потенциала" $\Omega$ вообще не зависит. Поэтому область локализации волновой функции в этом режиме есть $O(\hbar / \pi)$ вне зависимости от $\Omega$, а с ростом $\Omega$ (при фиксированном $\hbar$ ) увеличивается только частота осцилляций и растет $|E|$, причем в пределе $\Omega \rightarrow \infty$ зависимость между $|E|$ и $\Omega$ стремится к линейной. Иными словами, качественно поведение решения уравнения (1.1) как функции $\Omega$ при фиксированном $\hbar$ выглядит следуюшим образом. Пока $\Omega$ не слишком велико, так что выполняется неравенство (1.9), скорость убывания волновой функции при $|x|>\hbar / \pi$ монотонно увеличивается, далее при $\hbar \Omega=2 \pi$ она достигает максимума, после чего в области (1.15) происходит смена режима - область локализации больше не меняется, а на экспоненциальное убывание волновой функции накладываются осцилляции, частота которых растет с ростом $\Omega$. Уровень энергии при этом постоянно опускается вниз, выходя на линейную асимптотику при $\Omega \rightarrow \infty$. В то же время если в области (1.15) перейти к пределу $\hbar \rightarrow 0$ при фиксированном $\hbar \Omega$, то $|E|$ растет как $O\left(1 / \hbar^{2}\right)$, а период осцилляций и область локализации убывают как $O(\hbar)$, поэтому в этом случае у волновой функции 
вообще нет аналитического предела. Тем самым ничего похожего на решение уравнения (1.5) в этой области значений $\hbar$ и $\Omega$ мы не находим. Таким образом, окончательный вывод состоит в том, что в рамках конечно-разностной задачи (1.1) мы сталкиваемся с ситуацией, когда свойства решения сушественно зависят от соотношения между $ћ$ и параметрами потенциала $V(x)$, причем вполне возможна ситуация, когда при $\hbar \ll 1$ решение уравнения (1.1) тем не менее не будет иметь ничего обшего с решением уравнения (1.5).

Случай $\delta$-образного потенциала (1.6) является в определенном смысле исключительным, так как для него уравнение (1.1) имеет для всех значений $\Omega$ явное точное решение, с помощью которого можно наглядно продемонстрировать принципиальную разницу между уравнением (1.1) и его нерелятивистским дифференциальным аналогом (1.5). Физическое содержание уравнения (1.1) с таким потенциалом, однако, достаточно ограничено. Гораздо больший интерес с точки зрения фозических приложений представляет исследование уравнения (1.1) для случая прямоугольной потенциальной ямы, когда

$$
V(x)=-V_{0} \theta(a-|x|), \quad V_{0}>0
$$

В случае такого потенциала решение уравнения (1.1), в отличие от уравнения Шредингера (1.5), представляет отдельную нетривиальную проблему, исследование которой является основным содержанием настоящей работы. В частности, мы покажем, что, как и в случае с $\delta$-образным потенциалом, свойства решений уравнения (1.1) и статус предельного перехода от (1.1) к (1.5) будут сушественно зависеть от соотношения между $V_{0}$ и $\hbar$. Более того, поведение уровней энергии и волновых функций при (почти) неограниченном росте $V_{0}$ снова оказывается нетривиальным, равно как и физическая интерпретация этого эффекта.

В заключение этого раздела отметим следующее общее обстоятельство. Пример с $\delta$-образным потенциалом показывает, что прямолинейное разложение разностного оператора в уравнении (1.1) по степеням $\hbar^{2}$, которое приводит к представлению уравнения (1.1) в виде соответствующего ряда, начинающегося с уравнения Шредингера (1.5), может не иметь смысла и для $\hbar \ll 1$. Более того, такая теория возмущений оказывается непригодна даже для построения приближенных решений уравнения (1.1). Причина этого состоит в том, что обрывание этого ряда на конечном члене эквивалентно замене экспоненты (оператора конечного сдвига) на полином. Однако полином не дает равномерной аппроксимации экспоненты на всей числовой прямой, в результате чего решения обрезанного таким образом уравнения не будут иметь ничего обшего с истинными. Поэтому решение уравнения (1.1) в рамках разложения по степеням $\hbar^{2}$ требует суммирования всего ряда, что практически невыполнимо. Таким образом, построение даже приближенных решений уравнения (1.1) требует использования других методов, которые и будут рассмотрены в настояшей работе.

\section{2. ОБШАЯ ФОРМУЛИРОВКА КОНЕЧНО-РАЗНОСТНОЙ ЗАДАЧИ В СЛУЧАЕ ПРЯМОУГОЛЬНОЙ ПОТЕНЦИАЛЬНОЙ ЯМЫ}

Наиболее эффективный подход к решению уравнения (1.1) с потенциалом (1.21), равно как и с любыми другими кусочно-постоянными потенциалами, аналогичен решению 
соответствуюшего уравнения Шредингера: сначала уравнение (1.1) решается в областях постоянства $V(x)$, после чего полученные решения сшиваются между собой так, чтобы обеспечить аналитичность решения во всей полосе $|\operatorname{Im} z|<\hbar$. При этом, как и для уравнения (1.5), необходимым условием такой сшивки посредством аналитического продолжения оказывается уравнение, определяюшее уровни энергии.

Прежде чем приступить к детальному рассмотрению этой процедуры, удобно провести редукцию задачи по четности, так как в силу симметрии потенциала (1.21) все собственные функции уравнения (1.1) делятся на четные и нечетные. Кроме того, из действительности потенциала $V(x)$ и структуры разностного оператора в (1.1) следует, что функции $\psi(x)$ могут быть выбраны действительными. Поэтому далее без потери обшности мы можем ограничиться рассмотрением четных действительных решений уравнения $(1.1)$

$$
\psi(x)=\psi(-x)=\psi^{*}(x) .
$$

Перенос всех полученных результатов на нечетный случай будет тривиален.

Далее используем то обстоятельство, что для потенциала (1.21) уровни энергии связанных состояний уравнения (1.1), как и для уравнения (1.5), будут лежать в интервале $-V_{0}<E<0$, и введем удобную для дальнейших выкладок параметризацию. В частности, перейдем от $|E|$ к параметру $\varkappa$, введенному в (1.12), причем эта параметризация распространяется и на область $|E|>4 / \hbar^{2}$, если считать, что параметр $\varkappa$ при этом становится комплексным по правилу (1.20). Помимо $\varkappa$ нам потребуется ввести еше один параметр $\omega$, определенный соотношением

$$
V_{0}-|E|=\bar{E}=\frac{2}{\hbar^{2}}(\operatorname{ch} \hbar \omega-1)=\frac{4}{\hbar^{2}} \operatorname{sh}^{2}\left(\frac{\hbar \omega}{2}\right), \omega>0 .
$$

Заметим, что величина $\bar{E}$ есть положение уровня относительно дна ямы, поэтому она находится в интервале $0<\bar{E}<V_{0}$ и всегда положительна, а параметр $\omega$ тем самым всегда действителен. Кроме того, параметры $\varkappa$ и $\omega$ не независимы, а связаны друг с другом соотношением

$$
\operatorname{sh}^{2}\left(\frac{\hbar \omega}{2}\right)+\sin ^{2}\left(\frac{\hbar \varkappa}{2}\right)=\hbar^{2} \frac{V_{0}}{4} .
$$

Процедура построения аналитической функции $\psi(x)$ из решений уравнения $(1.1)$ в областях постоянства потенциала состоит из двух этапов. Сначала, пользуясь известными теоремами исчисления конечных разностей [7], находим для этих областей обшие решения, которые являются рядами Дирихле следующей структуры. Внутри ямы

$$
\psi(x)=\sum_{n=-\infty}^{+\infty} A_{n} \cos \omega_{n} x, \quad|x|<a,
$$

где

$$
i \omega_{n}=i \omega+\frac{2 \pi n}{\hbar}
$$

при этом в силу действительности $\psi(x)$

$$
A_{n}^{*}=A_{-n} .
$$


Вне ямы

$$
\psi(x)=\sum_{s=0}^{+\infty}\left(B_{s} e^{-\varkappa_{s}|x|}+\bar{B}_{s} e^{-\bar{\varkappa}_{s}|x|}\right), \quad|x|>a
$$

где

$$
\varkappa_{s}=\varkappa+\frac{2 \pi s}{\hbar}, \quad \bar{\varkappa}_{s}=\varkappa+\frac{2 \pi s}{\hbar},
$$

при этом если $|E|<4 / \hbar^{2}$, то $\varkappa$ действительное, и тогда

$$
\bar{\varkappa}=\frac{2 \pi}{\hbar}-\varkappa
$$

а коэффициенты $B_{s}, \bar{B}_{s}$ действительны и независимы, а если $|E|>4 / \hbar^{2}$, то $\varkappa$ комплексное и имеет вид (1.20), тогда

$$
\bar{\varkappa}=\frac{2 \pi}{\hbar}-\varkappa=\varkappa^{*}=\frac{\pi}{\hbar}-i \alpha,
$$

при этом коэффициенты $B_{s}, \bar{B}_{s}$ также комплексные, но в силу действительности $\psi(x)$ связаны друг с другом соотношением

$$
B_{s}^{*}=\bar{B}_{s}
$$

На следуюшем этапе коэффишиенты $A_{n}$ и $B_{s}, \bar{B}_{s}$ должны быть связаны таким образом, чтобы функции (2.4) и (2.7) аналитически продолжались друг в друга через отрезки $( \pm a-i \hbar, \pm a+i \hbar)$. Заметим, что вопрос о сходимости этих рядов в соответствуюших областях рассматривать специально не требуется, так как она автоматически следует из сходимости рядов на этих отрезках (в силу известных свойств рядов Дирихле достаточно сходимости в одной точке на каждом отрезке), которая с необходимостью будет проверяться при аналитическом продолжении. Кроме того, в силу четности рассматриваемого решения (2.1) достаточно обеспечить сходимость только на одном отрезке $(a-i \hbar, a+i \hbar)$

Прямолинейный способ аналитического продолжения состоит в сшивке этих рядов по непрерывности на указанном отрезке. Сушествует, однако, более эффективный способ обеспечить требуемый результат, преимушество которого состоит в том, что он практически сразу приводит к уравнениям, которые представляют из себя необходимое и достаточное условия аналитического продолжения и содержат только либо коэффициенты $A_{n}$, либо $B_{s}, \bar{B}_{s}$. Для этого запишем уравнение (1.1) в виде

$$
\frac{1}{\hbar^{2}}[\psi(x-i \hbar)+\psi(x+i \hbar)-2 \psi(x)]+|E| \psi(x)=V_{0} \theta(a-|x|) \psi(x)
$$

и с помощью функции Грина (1.11) перейдем от (2.12) к интегральному уравнению

$$
\psi(x)=V_{0} \int_{-a}^{a} d y G(x-y) \psi(y) .
$$

Очевидно, что при подстановке в (2.13) ряда (2.4) это уравнение должно быть тождеством при $|x|<a$. Более того, поскольку функция $G(z)$ аналитически продолжается на 
полосу $|\operatorname{Im} z|<\hbar$, это тождество должно иметь место в прямоугольнике $|\operatorname{Re} z|<a$, $|\operatorname{Im} z|<\hbar$. Из этих условий после некоторых преобразований находим искомые уравнения на коэффициенты $A_{n}$ в следуюшем виде:

$$
\begin{aligned}
& \sum_{n} A_{n}\left(\frac{e^{i \omega_{n} a}}{\varkappa_{s}-i \omega_{n}}+\frac{e^{-i \omega_{n} a}}{\varkappa_{s}+i \omega_{n}}\right)=0, \\
& \sum_{n} A_{n}\left(\frac{e^{i \omega_{n} a}}{\bar{\varkappa}_{s}-i \omega_{n}}+\frac{e^{-i \omega_{n} a}}{\bar{\varkappa}_{s}+i \omega_{n}}\right)=0,
\end{aligned}
$$

которые должны выполняться для любого $s, 0 \leqslant s \leqslant+\infty$. Заметим, что если $\varkappa$ действительное, то уравнения (2.14) и (2.14а) представляют из себя две различные системы действительных уравнений, которые должны решаться совместно. Если же $\varkappa$ комплексное, то уравнения систем также становятся комплексными, но при этом системы (2.14) и (2.14a) комплексно-сопряжены друг другу, поэтому в этом случае достаточно рассматривать только одну из них.

Если же рассмотреть уравнение (2.12) при $|x|>a$, то при подстановке в его правую часть ряда (2.4) получим выражения для $B_{s}, \bar{B}_{s}$ через $A_{n}$, которые обеспечивают требуемое аналитическое продолжение функции (2.4) в функцию (2.7):

$$
\begin{aligned}
& B_{s} e^{-\varkappa_{s} a}=\frac{\hbar V_{0}}{4 \sin \hbar \varkappa} \sum_{n} A_{n}\left(\frac{e^{i \omega_{n} a}}{\varkappa_{s}+i \omega_{n}}+\frac{e^{-i \omega_{n} a}}{\varkappa_{s}-i \omega_{n}}\right), \\
& \bar{B}_{s} e^{-\bar{\varkappa}_{s} a}=\frac{\hbar V_{0}}{4 \sin \hbar \bar{\varkappa}} \sum_{n} A_{n}\left(\frac{e^{i \omega_{n} a}}{\bar{\varkappa}_{s}+i \omega_{n}}+\frac{e^{-i \omega_{n} a}}{\bar{\varkappa}_{s}-i \omega_{n}}\right) .
\end{aligned}
$$

Эту же операцию целесообразно проделать еше раз, переписав исходное уравнение (1.1) в виде

$$
\bar{E} \psi(x)-\frac{1}{\hbar^{2}}[\psi(x-i \hbar)+\psi(x+i \hbar)-2 \psi(x)]=V_{0} \theta(|x|-a) \psi(x)
$$

и снова перейдя к интегральной форме

$$
\psi(x)=V_{0}\left[\int_{-\infty}^{-a}+\int_{a}^{\infty}\right] d y \bar{G}(x-y) \psi(y)
$$

где $\bar{G}(x)$ имеет вид

$$
\bar{G}(x)=\frac{\hbar}{2 \operatorname{sh} \hbar \omega} \frac{\sin \omega x}{\operatorname{th}(\pi x / \hbar)} .
$$

Повторяя рассуждения, использованные при переходе от (2.13) к (2.14), (2.15), из (2.17) при $|x|>a$ получим уравнение на коэффициенты $B_{s}, \bar{B}_{s}$

$$
\sum_{s=0}^{\infty}\left[B_{s} e^{-\varkappa_{s} a}\left(\frac{e^{i \omega_{n} a}}{\varkappa_{s}-i \omega_{n}}+\frac{e^{-i \omega_{n} a}}{\varkappa_{s}+i \omega_{n}}\right)+\bar{B}_{s} e^{-\bar{\varkappa}_{s} a}\left(\frac{e^{i \omega_{n} a}}{\bar{\varkappa}_{s}-i \omega_{n}}+\frac{e^{-i \omega_{n} a}}{\bar{\varkappa}_{s}+i \omega_{n}}\right)\right]=0
$$


которое должно выполняться для любого $n,-\infty \leqslant n \leqslant+\infty$, а при $|x|<a$ - выражения для $A_{n}$ через $B_{s}, \bar{B}_{s}$, обратные к $(2.15)$ :

$$
\begin{aligned}
A_{n} & =\frac{\hbar V_{0}}{i \operatorname{sh} \hbar \omega} e^{i \omega_{n} a} \sum_{s=0}^{\infty}\left(\frac{B_{s} e^{-\varkappa_{s} a}}{\varkappa_{s}-i \omega_{n}}+\frac{\bar{B}_{s} e^{-\bar{\varkappa}_{s} a}}{\bar{\varkappa}_{s}-i \omega_{n}}\right)= \\
& =-\frac{\hbar V_{0}}{i \operatorname{sh} \hbar \omega} e^{-i \omega_{n} a} \sum_{s=0}^{\infty}\left(\frac{B_{s} e^{-\varkappa_{s} a}}{\varkappa_{s}+i \omega_{n}}+\frac{\bar{B}_{s} e^{-\bar{\varkappa}_{s} a}}{\bar{\varkappa}_{s}+i \omega_{n}}\right) .
\end{aligned}
$$

Заметим, что появление двух различных выражений для $A_{n}$ в (2.20) есть следствие того, что $A_{n}$ удовлетворяют условию (2.6), а уравнение (2.19) при этом может быть получено непосредственно приравниванием этих двух выражений для $A_{n}$.

Таким образом, решение исходного уравнения (1.1) с потенциалом (1.21) оказывается эквивалентно одной из бесконечных однородных систем (2.14), (2.14а) или (2.19) на коэффишиенты $A_{n}$ или $B_{s}, \bar{B}_{s}$, формальное условие разрешимости которых есть равенство нулю соответствуюшего детерминанта, из которого в конечном счете и определяются уровни энергии в этой задаче. Сложность проблемы состоит в том, что из-за наличия экспоненциальных множителей $e^{ \pm 2 \pi n a / \hbar}$ в коэффициентах системы $(2.14),(2.14 \mathrm{a})$ и (2.19) не принадлежат ни к одному из исследованных аналитическими или численными методами классов бесконечных линейных систем [9], [10]. Как следствие общих методов решения подобных систем в настояшее время не существует. В этой работе мы покажем, однако, что для систем $(2.14),(2.14 \mathrm{a})$ и (2.19) сушествует ряд специальных приемов, которые позволяют получить достаточно полную информацию о спектре собственных значений и свойствах волновых функций в широком диапазоне изменения параметров задачи.

\section{3. ТЕОРИЯ ВОЗМУЩЕНИЙ ПО ћ ДЛЯ “НЕГЛУБОКОЙ ЯМЫ"}

В качестве первого метода исследования систем (2.14), (2.14a) и (2.19) рассмотрим их решение в рамках теории возмушений по $\hbar$ в окрестности соответствуюшего решения уравнения Шредингера (1.5). Эта теория возмушений, однако, сушественно отличается от прямолинейного разложения исходного уравнения (1.1) в ряд по $\hbar^{2}$, о котором упоминалось в конце раздела 1 , и эффективно работает для $\hbar \ll 1$ и достаточно неглубокой ямы. Эти условия более точно формулируются следующим образом:

$$
\varkappa, \omega \simeq O(1) \ll \frac{1}{\hbar},
$$

при этом соотношение (2.3) принимает вид

$$
\varkappa^{2}+\omega^{2}=V_{0}
$$

Таким образом, глубина ямы $V_{0}$ в данном случае должна быть такова, чтобы удовлетворять соотношениям, аналогичным (3.1), а именно

$$
V_{0} \simeq O(1) \ll \frac{1}{\hbar}
$$


Сам по себе метод решения систем $(2.14),(2.14 a)$ и (2.19) в рамках теории возмушений основан на том обстоятельстве, что нулевое приближение в терминах коэффициентов $A_{n}$ и $B_{s}, \bar{B}_{s}$ задается максимально простым образом. Предварительно заметим, что для сходимости рядов $(2.4)$ и $(2.7)$ на отрезке $(a-i \hbar, a+i \hbar)$ коэффициенты $A_{n}$ должны убывать при $|n| \rightarrow \infty$ заведомо быстрее, чем $e^{-2 \pi|n| a / \hbar}$, а $B_{s}, \bar{B}_{s}$ при $s \rightarrow \infty$ - возрастать медленнее, чем $e^{2 \pi s a / \hbar}$. Тогда в рамках теории возмущений по $\hbar$ коэффициенты $A_{0}, B_{0}$ следует рассматривать как величины, соответствуюшие нулевому приближению, которое мы для удобства будем считать порядка $O(1)$, а остальные $A_{n} e^{2 \pi|n| a / \hbar}$, $n \neq 0, B_{s} e^{-2 \pi s a / \hbar}, s \neq 0$, и все $\bar{B}_{s} e^{-2 \pi s a / \hbar}$ - как поправки следующего порядка малости, т.е. в данном случае порядка $O(\hbar)$. Чтобы убедиться в этом, заметим, что при таких условиях нулевое $O(1)$-приближение в рядах $(2.4)$ и (2.7) воспроизводит структуру четного решения уравнения Шредингера (1.5), а уравнение с $s=0$ системы (2.14) и уравнение с $n=0$ системы (2.19) сводятся к соотношениям

$$
A_{0}\left(\frac{e^{i \omega a}}{\varkappa-i \omega}+\frac{e^{-i \omega a}}{\varkappa+i \omega}\right)=0, \quad B_{0}\left(\frac{e^{i \omega a}}{\varkappa-i \omega}+\frac{e^{-i \omega a}}{\varkappa+i \omega}\right)=0 .
$$

Поскольку коэффициенты $A_{0}, B_{0}$ заведомо не нулевые, то в (3.4) должен обрашаться в нуль множитель при них, что дает

$$
\frac{\varkappa}{\omega}=\operatorname{tg} \omega a .
$$

Это соотношение совместно с (3.2) суть не что иное, как уравнения, определяюшие четные уровни энергии в уравнении Шредингера (1.5). В свою очередь, коэффициенты $A_{0}$, $B_{0}$, которые остаются произвольными после решения системы (3.4), приобретают вполне определенный смысл амплитуд шредингеровской волновой функции внутри и вне ямы и тем самым действительно имеют статус величин нулевого приближения.

После того как зафиксировано нулевое приближение и установлена иерархия порядков по $\hbar$ для коэффициентов $A_{n}$ и $B_{s}, \bar{B}_{s}$, решение систем $(2.14),(2.14 \mathrm{a})$ и $(2.19)$ по теории возмушений происходит в рамках стандартного рекурсивного процесса. Поскольку системы $(2.14),(2.14 \mathrm{a})$ и (2.19) с точки зрения решения исходного уравнения (1.1) эквивалентны, рассмотрим этот метод на примере решения систем $(2.14),(2.14 \mathrm{a})$ (в данном

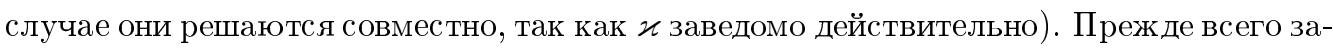
метим, что из условия (3.1) следует, что знаменатели коэффициентов системы (2.14), равные $\varkappa_{s} \pm i \omega_{n}=\varkappa \pm i \omega+2 \pi(s \pm n) / \hbar$, содержат два слагаемых разного порядка по $\hbar$, именно $|\varkappa \pm i \omega| \ll|2 \pi(s \pm n) / \hbar|$, если только $s \pm n \neq 0$. Аналогичное разбиение имеется и в знаменателях коэффициентов системы (2.14a), только при этом следует учесть, что теперь $\bar{\varkappa}=2 \pi / \hbar-\varkappa \simeq 2 \pi / \hbar$. Последнее обстоятельство означает, что в рамках рассматриваемой схемы системы (2.14) и (2.14a) не равноценны, и между отдельными уравнениями этих систем сушествует определенная иерархия: одинаковый статус будут иметь уравнения с номером $s$ из (2.14) и $s-1$ из (2.14а). Уравнение с $s=0$ из (2.14) при этом играет особую роль, поскольку задает нулевое приближение и, следовательно, партнера в (2.14a) не имеет.

Чтобы найти первое приближение для коэффициентов $A_{n}, \quad n \neq 0$, рассмотрим последовательно пары уравнений с номерами $s$ из (2.14) и $s-1$ из (2.14а) для всех $s>0$. Легко видеть, что старшие члены в этих уравнениях имеют порядок величины $O(\hbar)$ и 
соответствуют слагаемым с $n=0, n= \pm s$. Кроме того, если яма не является предельно узкой (здесь и далее под предельно узкой ямой понимается случай $a \leqslant \hbar$ ), то для всех $n \neq 0$ справедливо неравенство $e^{-2 \pi a|n| / \hbar} \ll 1$, что позволяет в множителях при $A_{ \pm s}$ оставить только по одному из двух слагаемых. В результате для каждого номера $s>0$ имеем по два уравнения для определения коэффициентов $A_{ \pm s}$ :

$$
\begin{aligned}
& A_{s} \frac{e^{i \omega a}}{\varkappa-i \omega}+A_{-s} \frac{e^{-i \omega a}}{\varkappa+i \omega}=-A_{0} \frac{\hbar \cos \omega a}{\pi s} e^{-2 \pi s a / \hbar} \\
& A_{s} \frac{e^{i \omega a}}{\varkappa+i \omega}+A_{-s} \frac{e^{-i \omega a}}{\varkappa-i \omega}=A_{0} \frac{\hbar \cos \omega a}{\pi s} e^{-2 \pi s a / \hbar}
\end{aligned}
$$

откуда

$$
A_{ \pm s}= \pm A_{0} \frac{i \hbar V_{0}}{\pi s} \frac{e^{\mp i \omega a} \cos \omega a}{2 \omega} e^{-2 \pi s a / \hbar}, \quad s>0,
$$

при этом из уравнений (3.7) получаем $A_{s}^{*}=A_{-s}$ в соответствии с (2.6). Кроме того, найденные таким образом коэффициенты $A_{n}$ убывают как $e^{-2 \pi|n| a / \hbar} /|n|$ при $|n| \rightarrow \infty$, что полностью оправдьвает сделанное ранее предположение о характере их убывания. Заметим также, что для правильного порядка малости коэффициентов $A_{n} e^{2 \pi|n| a / \hbar}, n \neq 0$, глубина ямы $V_{0}$ должна обязательно удовлетворять соотношению (3.3), т.е. для обоснования теории возмущений в окрестности решения уравнения (1.5) условие неглубокой ямы является не менее сушественным, чем малость $\hbar$. В результате повторяется ситуация, описанная в разделе 1 для $\delta$-образного потенциала, когда для возможности предельного перехода точного решения уравнения (1.1) в решение соответствуюшего уравнения Шредингера при $\hbar \rightarrow 0$ должно было выполняться одновременно и ограничение сверху на величину потенциала.

Из соотношения (3.7) также следует, что за счет множителей $e^{-2 \pi|n| a / \hbar}$ в коэффициентах $A_{n}$ поправки к шредингеровской волновой функции, которые возникают за счет вклада от членов с $n \neq 0$ в ряде $(2.4)$, будут сушественны только в $O(\hbar / \pi)$-окрестности точек $x= \pm a$. Как и в случае $\delta$-образного потенциала, эта окрестность точек $x= \pm a$ представляет из себя переходную область, где за счет поправок от членов с $n \neq 0$ обеспечивается более гладкий, чем у нулевого приближения, переход от решения внутри ямы к решению вне ямы.

Итерируя системы $(2.14),(2.14 a)$ далее, мы получим сходяшуюся для достаточно малых $\hbar$ теорию возмушений в окрестности решения уравнения Шредингера (1.5), имеющую своим пределом точное решение уравнения (1.1) с требуемыми аналитическими свойствами, которое будет отличаться от шредингеровской волновой функции только в переходной области размером $O(\hbar / \pi)$ в окрестности точек $x= \pm a$ степенью гладкости перехода от внутреннего решения к внешнему. Вне этих областей вклад в ряд (2.4) от слагаемых с $n \neq 0$ будет пренебрежимо мал за счет множителей $e^{-2 \pi|n|(a-|x|) / \hbar}$, а при $\hbar \rightarrow 0$ он исчезнет полностью, и в ряде $(2.4)$ останется только член $A_{0} \cos \omega x$, соответствуюший решению уравнения (1.5).

Отметим также два существенных обстоятельства, возникающих в такой теории возмушений. Первое касается свойств второй итерации, первым шагом которой является уточнение уравнения с $s=0$ в системе (2.14) при подстановке в него найденных в (3.7) коэффициентов $A_{n}, n \neq 0$. Эта подстановка должна приводить к поправке порядка $O\left(\hbar^{2}\right)$ 
к уравнению нулевого приближения (3.5) на уровни энергии. Легко видеть, однако, что эта поправка тождественно равна нулю, по крайней мере если яма не является предельно узкой. Тогда из множителей при $A_{n}$ можно оставить только одно слагаемое, соответствуюшее растущей экспоненте, в результате чего эта поправка имеет вид

$$
\sum_{n \geqslant 1} A_{n} \frac{e^{i \omega_{n} a}}{\varkappa-i \omega_{n}}+\sum_{n \leqslant-1} A_{n} \frac{e^{-i \omega_{n} a}}{\varkappa+i \omega_{n}}
$$

а после подстановки выражений (3.7) для $A_{n}$ и упрошения знаменателей получаем

$$
-i \hbar^{2} \frac{V_{0} \cos \omega a}{4 \pi^{2} \omega} A_{0}\left(\sum_{n \geqslant 1} \frac{1}{n^{2}}-\sum_{n \leqslant-1} \frac{1}{n^{2}}\right)=0 .
$$

Таким образом, в рассматриваемой теории возмушений по степеням $\hbar$ поправки к шредингеровским уровням энергии начинаются только с порядка $O\left(\hbar^{4}\right)$, так как в силу симметрии уравнения (1.1) относительно знака $\hbar$ энергия уровня должна представляться рядом, содержащим только четные степени $\hbar$. Более того, можно заранее утверждать, что в целом поправки к шредингеровским уровням будут сдвигать их вверх. Причина этого состоит в том, что в рамках теории возмушений шредингеровская и точная волновые функции отличаются только в переходных областях размером $O(\hbar)$ по степени гладкости, поэтому имеют практически одинаковую потенциальную энергию. С другой стороны, при одной и той же функции значение функционала (1.4), определяющего согласно (1.1a) кинетический вклад в энергию для решений уравнения (1.1), будет заведомо больше, чем кинетическая энергия в шредингеровском случае.

Второе обстоятельство связано с тем, что ряд (2.4) с коэффициентами $A_{n}$ вида $(3.7)$ сходится во всех внутренних точках отрезка $(a-i \hbar, a+i \hbar)$, как и требуется по условию, но логарифмически расходится в точках $a \pm i \hbar$. Иными словами, функции, определяемые рядами (2.4) и (2.7), имеют при $z=a \pm i \hbar$ логарифмические точки ветвления, а функция $\psi(z)$, возникаюшая в результате сшивки рядов на отрезке $(a-i \hbar, a+i \hbar)$, обладает следуюшими аналитическими свойствами: в силу свойств рядов Дирихле она аналитична всюду при $|\operatorname{Re} z| \neq a$, а при $|\operatorname{Re} z|=a$ имеет вертикальные разрезы, начинающиеся в точках $\pm a+i \hbar, \pm a-i \hbar$.

Последнее утверждение верно и вне рамок теории возмущений, что легко подтвердить следуюшими рассуждениями. Запишем $\psi(z)$ внутри и вне ямы в правой полуплоскости следуюшим образом:

$$
\begin{aligned}
\psi_{\text {in }}(z) & =A(z) e^{i \omega z}+A^{*}\left(z^{*}\right) e^{-i \omega z}, & |\operatorname{Re} z|<a, \\
\psi_{\text {out }}(z) & =B(z) e^{-\varkappa z}+\bar{B}(z) e^{-\bar{\varkappa} z}, & \operatorname{Re} z>a,
\end{aligned}
$$

где

$$
A(z)=\frac{1}{2} \sum_{n} A_{n} e^{2 \pi n z / \hbar}, \quad B(z)=\sum_{s} B_{s} e^{-2 \pi s z / \hbar}, \quad \bar{B}(z)=\sum_{s} \bar{B}_{s} e^{-2 \pi s z / \hbar}
$$

суть $i \hbar$-периодические функции. Поэтому если предположить, что ряды (3.12) сходятся всюду на отрезке $[a, a+i \hbar]$, включая граничные точки, то они сходятся и на всей прямой 
$(a-i \infty, a+i \infty)$. Тогда функции $\psi_{\text {in }}$ и $\psi_{\text {out }}$ будут аналитичны всюду при $|\operatorname{Re} z| \leqslant a$ и $\operatorname{Re} z \geqslant a$, соответственно, а возникаюшая после их сшивки через отрезок $(a-i \hbar, a+i \hbar)$ функция $\psi(z)$ будет целой во всей полуплоскости $\operatorname{Re} z>-a$. В силу единственности аналитического продолжения это означает, что $\psi_{\text {in }}$ и $\psi_{\text {out }}$ должны совпадать на всей вертикальной прямой $(a-i \infty, a+i \infty)$. Это условие удобно представить как $\psi_{\text {in }}(z)=$ $\psi_{\text {out }}(z)$ для $z=z_{0}+i n \hbar$, где $n$ целое, $\mathrm{a} z_{0} \in[a, a+i \hbar]$, что приводит к следующей системе равенств:

$$
\mathcal{A}_{0} e^{-n \hbar \omega}+\overline{\mathcal{A}}_{0} e^{n \hbar \omega}=\mathcal{B}_{0} e^{-i n \hbar \varkappa}+\overline{\mathcal{B}}_{0} e^{i n \hbar \bar{\varkappa}}, \quad-\infty \leqslant n \leqslant+\infty,
$$

где

$$
\begin{array}{ll}
\mathcal{A}_{0}=A\left(z_{0}\right) e^{i \omega z_{0}}, & \overline{\mathcal{A}}_{0}=A^{*}\left(z_{0}^{*}\right) e^{-i \omega z_{0}}, \\
\mathcal{B}_{0}=B\left(z_{0}\right) e^{-\varkappa z_{0}}, & \overline{\mathcal{B}}_{0}=\bar{B}\left(z_{0}\right) e^{-\bar{\varkappa} z_{0}} .
\end{array}
$$

Взяв любые три последовательных уравнения (3.13), после несложных вычислений получим условие их совместности в виде

$$
\left(\mathcal{A}_{0} e^{-n \hbar \omega}+\overline{\mathcal{A}}_{0} e^{n \hbar \omega}\right)[\operatorname{ch} \hbar \omega-\cos \hbar \varkappa]=0 .
$$

Это уравнение разрешимо либо в виде

$$
\varkappa=\omega=0,
$$

что в силу (2.3) эквивалентно $V_{0}=0$ и поэтому невозможно, либо в виде

$$
\mathcal{A}_{0} e^{-n \hbar \omega}+\overline{\mathcal{A}}_{0} e^{n \hbar \omega}=0
$$

Последнее условие в силу произвольности $z_{0}$ и $n$ означает, что $\psi(z)=0$, если $z \in(a-$ $i \infty, a+i \infty)$, но тогда $\psi(z) \equiv 0$ всюду. Полученное противоречие показывает, что ряды (3.12) обязательно должны иметь особые точки на конщах отрезка $[a, a+i \hbar]$. При этом за счет "игры" коэффициентов в $\psi_{\text {in }}(a)$ и $\psi_{\text {out }}(a)$ эти расходимости сокрашаются, как, например, это происходит при подстановке коэффициентов (3.7) в ряд (2.4), поэтому функция $\psi(z)$ оказывается аналитической во всей полосе $|\operatorname{Im} z|<\hbar$, но в точках $\pm a+i n \hbar, n \neq 0$, такого сокрашения не происходит, и поэтому эти точки являются точками ветвления $\psi(z)$.

Заметим, что схожим образом ведет себя и решение уравнения (1.1) для $\delta$-образного потенциала, меняется лишь тип особых точек. Именно, функции (1.13) и (1.18) конечны при $x=0$, но имеют простые полюсы при $z=i n \hbar, n \neq 0$. Таким образом, особые точки на прямых $|\operatorname{Im} z|=\hbar$ и разрезы, идушие от этих точек, в решениях уравнения (1.1) являются следствием разрывов в потенциале. Поэтому следует ожидать, что для более гладких потенциалов $V(x)$ (по крайней мере, для аналитических в полосе $|\operatorname{Im} z|<\hbar$ ) таких особых точек не будет, хотя строгое доказательство этого утверждения на данньй момент отсутствует. 


\section{4. КВАЗИТОЧНОЕ АНАЛИТИЧЕСКОЕ РЕШЕНИЕ ЗАДАЧИ ДЛЯ “ШИРОКОЙ ЯМЫ"}

Рассмотренная в предыдушем разделе теория возмушений в окрестности решения уравнения Шредингера (1.5), несмотря на свою простоту и эффективность при малых $\hbar$, применима в весьма ограниченном диапазоне изменения параметров задачи. Для получения надежной информации о спектре и собственных функциях уравнения (1.1) для значений параметров, выходящих за рамки условий (3.1)-(3.3), требуются качественно другие методы, один из которых изложен в настояшем разделе. Основным условием применимости этого метода является достаточная ширина ямы $a$, при которой выполняется условие

$$
\lambda=e^{-2 \pi a / \hbar} \ll 1 .
$$

Заметим, что это условие является физически вполне допустимым, так как для его выполнения достаточно считать $a \geqslant \hbar$, поскольку уже при $a=\hbar$ имеем $\lambda=e^{-2 \pi a / \hbar}=$ $e^{-2 \pi} \simeq 10^{-3}$. Никаких других условий, кроме (4.1), первоначальная формулировка метода не требует, хотя в дальнейшем при проверке соответствия полученных результатов истинному решению появится и ряд других ограничений на параметры задачи, которые, однако, будут сушественно более слабыми.

Как и в случае теории возмущений по $\hbar$, с помощью этого метода можно с одинаковой эффективностью решать как "внутреннюю" задачу $(2.14),(2.14 a)$ на коэфффициенты $A_{n}$, так и "внешнюю" систему (2.19). По соображениям удобства изложения мы будем рассматривать последнюю, так как в этом случае не нужно разбирать по отдельности случаи действительного и комплексного $\varkappa$.

Начнем с того, что определим следуюшую функцию комплексного переменного $z$ :

$$
f(z)=\sum_{s=0}^{\infty}\left(\frac{C_{s}}{z-\varkappa_{s}}+\frac{\bar{C}_{s}}{z-\bar{\varkappa}_{s}}\right)
$$

где в целях компактности дальнейших выкладок введены обозначения

$$
C_{s}=B_{s} e^{-\varkappa_{s} a}, \quad \bar{C}_{s}=\bar{B}_{s} e^{-\bar{\varkappa}_{s} a} .
$$

В терминах функции $f(z)$ система (2.19) записывается в виде

$$
e^{i \omega_{n} a} f\left(i \omega_{n}\right)+e^{-i \omega_{n} a} f\left(-i \omega_{n}\right)=0 .
$$

Заметим теперь, что в силу соотношений $(2.8)-(2.11)$ функция $f(z)$ удовлетворяет условию действительности на вешественной оси

$$
f\left(z^{*}\right)=f^{*}(z),
$$

а из определения $(2.5)$ следует, что $\left(i \omega_{n}\right)^{*}=-i \omega_{-n}$. С помошью этих соотношений легко видеть, что в системе (4.4), равно как и в (2.19), уравнения с номерами $\pm n$ комплексно-сопряжены друг другу, поэтому для решения этих систем достаточно уравнений с $n \geqslant 0$. 
В дальнейшем наиболее сушественным обстоятельством является условие (4.1), которое означает, что для $n>0$ второе слагаемое в (4.4) имеет мальй множитель $e^{-2 \pi n a / \hbar}=\lambda^{n}$. Поэтому возникает естественное предположение, что при $n>0$ второе слагаемое в (4.4) мало по сравнению с первым и может быть учтено как поправка в рамках разложения по степеням $\lambda$ (корректность этого предположения будет в обязательном порядке проверяться при анализе найденного таким образом решения). Нулевой порядок такого разложения - приближение "широкой ямы" - имеет для системы (4.4) при $n>0$ вид

$$
f\left(i \omega_{n}\right)=0 .
$$

Из условия (4.5) при этом следует, что функция $f(z)$ одновременно должна обрашаться в нуль при $z=\left(i \omega_{n}\right)^{*}=-i \omega_{-n}$, т.е. в этом приближении функция $f(z)$ имеет в правой полуплоскости нули, которые располагаются парами в точках

$$
z_{n}= \pm i \omega_{ \pm n}= \pm i \omega+\frac{2 \pi n}{\hbar}, \quad n>0 .
$$

Следует отметить, что появление $( \pm \omega)$ в нулях функции $f(z)$ имеет весьма простое объяснение: параметр $\omega$ вводится соотношением (2.2), в котором его знак не фиксируется и далее нигде не используется, а выбирается положительным лишь для однозначности определения. Поэтому оба знака $\omega$ абсолютно равноправны, и именно это обстоятельство находит свое отражение в нулях функции $f(z)$.

Покажем теперь, что помимо соотношений (4.5)-(4.7) функция $f(z)$ обладает рядом дополнительных свойств, позволяюших восстановить ее вид с точностью до постоянного множителя. Сначала учтем, что из условия конечности функции $\psi_{\text {out }}(z)$ при $z=a$ следует, что ряд

$$
\sum_{s}\left(C_{s}+\bar{C}_{s}\right)=\psi_{\text {out }}(a)
$$

сходится, тогда по признаку Абеля-Дирихле сходится и ряд $\sum_{s>0}\left(C_{s}+\bar{C}_{s}\right) / s . \mathrm{C}$ другой стороны, $\left(C_{s}+\bar{C}_{s}\right) / s$ есть старший член асимптотики ряда в правой части (4.2) для $s \rightarrow \infty$, поэтому ряд (4.2) сходится для всех $z \neq \varkappa_{s}, \bar{\varkappa}_{s}$. (Ниже мы подтвердим эти свойства сходимости явным вычислением асимптотик коэффициентов $C_{s}$ и $\bar{C}_{s}$.) Тем самым функция $f(z)$ является мероморфной с простыми полюсами в точках $\varkappa_{s}, \bar{\varkappa}_{s}$, причем

$$
f(z) \rightarrow \frac{\psi(a)}{z}, \quad|z| \rightarrow \infty,
$$

где $\psi(a)=\psi_{\text {in }}(a)=\psi_{\text {out }}(a)$ - значение волновой функции $\psi(x)$ при $x=a$. Отметим, что соотношение (4.9) верно для всех лучей, за исключением положительной полуоси (если $\varkappa$ действительно) и параллельных ей лучей с $\operatorname{Im} z= \pm \alpha($ если $\varkappa=\pi / \hbar \pm i \alpha)$, где лежат полюсы $f(z)$.

Предположим теперь, что функция $f(z)$ не имеет других нулей, кроме как в точках (4.7), и все эти нули простые. Тогда после приведения к общему знаменателю правой части уравнения (4.2) функция $f(z)$ будет представлять из себя отношение двух целых функций, знаменателем в котором будет бесконечное произведение вида $\prod_{s}\left(z-\varkappa_{s}\right)(z-$ $\left.\bar{\varkappa}_{s}\right)$. Легко видеть, что тогда числитель этого отношения должен быть бесконечным произведением, условное “число" сомножителей в котором на единицу меньше, чем в 
знаменателе $\mathrm{e}^{1)}$. При этом числитель должен обращаться в нуль в точках (4.7) и только в них, поэтому это произведение должно иметь вид $\prod_{n>0}\left(z-i \omega_{n}\right)\left(z+i \omega_{-n}\right)$. В результате находим $f(z)$ в виде

$$
f(z)=\frac{A}{z-\varkappa} \prod_{k \geqslant 0} \frac{\left(z-i \omega_{k+1}\right)\left(z+i \omega_{-(k+1)}\right)}{\left(z-\varkappa_{k+1}\right)\left(z-\bar{\varkappa}_{k}\right)},
$$

где внешний множитель $(z-\varkappa)^{-1}$ учитывает разницу “степеней" числителя и знаменателя. Заметим, что в качестве этого внешнего множителя можно было бы использовать любой другой сомножитель из $\prod_{s}\left(z-\varkappa_{s}\right)\left(z-\bar{\varkappa}_{s}\right)$, конечный результат от такой перестановки не изменится.

Легко проверить, что бесконечное произведение в (4.10) сходится. Более того, используя известное соотношение для Г-функций

$$
\frac{\Gamma(\alpha) \Gamma(\beta)}{\Gamma(\alpha+\gamma) \Gamma(\beta-\gamma)}=\prod_{k=0}^{\infty} \frac{k+\alpha+\gamma}{k+\alpha} \frac{k+\beta-\gamma}{k+\beta}
$$

можно получить для $f(z)$ следующие представления:

$$
\begin{aligned}
& f(z)=\frac{A}{z-\varkappa} \frac{\Gamma[1-\hbar(z-\varkappa) / 2 \pi] \Gamma[1-\hbar(z+\varkappa) / 2 \pi]}{\Gamma[1-\hbar(z-i \omega) / 2 \pi] \Gamma[1-\hbar(z+i \omega) / 2 \pi]}, \\
& f(z)=A^{\prime} \frac{\Gamma[-\hbar(z-\varkappa) / 2 \pi] \Gamma[-\hbar(z-\varkappa) / 2 \pi]}{\Gamma[1-\hbar(z-i \omega) / 2 \pi] \Gamma[1-\hbar(z+i \omega) / 2 \pi]},
\end{aligned}
$$

где $A^{\prime}=-\hbar A / 2 \pi$. Из условия (4.5) и соотношений $(2.9),(2.10)$ при этом следует, что $A$, $A^{\prime}$ - действительные множители,

$$
A=A^{*}, \quad A^{\prime}=A^{\prime *},
$$

причем как представления (4.12), (4.12a), так и соотношения (4.13) имеют место как для действительных, так и для комплексных значений $\varkappa$.

Далее из соотношения (4.12) находим (с учетом замечаний, сделанных после формулы $(4.9))$

$$
f(z) \rightarrow \frac{A}{z}, \quad|z| \rightarrow \infty .
$$

Из совпадения асимптотик (4.14) и (4.9) следует, что никаких других нулей, кроме как в точках (4.7), функция $f(z)$ иметь не может, причем все имеющиеся нули должны быть обязательно простыми. Действительно, любой дополнительный нуль в некоторой точке $\xi$ (или нуль кратности $>1$ в одной из точек $z_{n}$ ) добавляет к выражению (4.12) множитель $(z-\xi)\left(z-\xi^{*}\right)$. Это, в свою очередь, добавляет к асимптотике $f(z)$ дополнительньй

\footnotetext{
1) Строго говоря, решение бесконечной системы уравнений (4.4) можно рассматривать как предел решения конечной системы, когда последовательности $s$ и $n$ обрезаны сверху на некотором $N$, а уравнение на спектр - как предел условия совместности этой конечной системы при $N \rightarrow \infty$. Для конечной системы ряд (4.2) становится суммой конечного числа членов, для которой утверждение о соотношении числа сомножителей числителя и знаменателя очевидно.
} 
множитель $z^{2}$, что противоречит условию (4.9). В результате, как и было анонсировано, функция $f(z)$ оказьвается однозначно определенной с точностью до действительного множителя $A$.

Заметим теперь, что для определения $f(z)$ мы использовали только уравнения (4.4) с $n>0$, тогда как полный набор независимых уравнений системы (4.4) включает еше и уравнение с $n=0$. В последнем множители вида $e^{ \pm 2 \pi n a / \hbar}$ отсутствуют, поэтому одинаково значимыми являются оба слагаемых $e^{ \pm i \omega a} f( \pm i \omega)$. В результате после подстановки вместо $f( \pm i \omega)$ выражений (4.12) это уравнение принимает вид

$$
e^{2 i \omega a}=\frac{\Gamma(-i \hbar \omega / \pi) \Gamma[\hbar(\varkappa+i \omega) / 2 \pi] \Gamma[\hbar(\bar{\varkappa}+i \omega) / 2 \pi]}{\Gamma(i \hbar \omega / \pi) \Gamma[\hbar(\varkappa-i \omega) / 2 \pi] \Gamma[\hbar(\bar{\varkappa}-i \omega) / 2 \pi]},
$$

что представляет из себя уравнение на уровни энергии в рассматриваемом приближении "широкой ямы", когда в системе (4.4) для $n>0$ можно пренебречь слагаемым с убывающей экспонентой $e^{-2 \pi n a / \hbar}$.

Прежде чем детально рассмотреть уравнение (4.15) и область его применимости, уместно сначала остановиться на основных свойствах коэффициентов $A_{n}$ и $C_{s}, \bar{C}_{s}$ и волновой функции $\psi(x)$, которые соответствуют такому приближенному решению уравнения (1.1). Заметим, что при известной функции $f(z)$ коэффициенты $A_{n}$ и $C_{s}, \bar{C}_{s}$ находятся весьма просто. В частности, $C_{s}, \bar{C}_{s}$ являются вычетами $f(z)$ в полюсах,

$$
C_{s}=\left.\operatorname{Res}\right|_{z=\varkappa_{s}} f(z), \quad \bar{C}_{s}=\left.\operatorname{Res}\right|_{z=\bar{\varkappa}_{s}} f(z),
$$

и имеют следующий явный вид:

$$
\begin{aligned}
& C_{s}=A \frac{\sin [\hbar(\varkappa+i \omega) / 2] \sin [\hbar(\varkappa-i \omega) / 2]}{\pi \sin \hbar \varkappa} \frac{\Gamma[s+\hbar(\varkappa+i \omega) / 2 \pi] \Gamma[s+\hbar(\varkappa-i \omega) / 2 \pi]}{\Gamma(s+1) \Gamma(s+\hbar \varkappa / \pi)}, \\
& \bar{C}_{s}=A \frac{\sin [\hbar(\bar{\varkappa}+i \omega) / 2] \sin [\hbar(\bar{\varkappa}-i \omega) / 2]}{\pi \sin \hbar \bar{\varkappa}} \frac{\Gamma[s+\hbar(\bar{\varkappa}+i \omega) / 2 \pi] \Gamma[s+\hbar(\bar{\varkappa}-i \omega) / 2 \pi]}{\Gamma(s+1) \Gamma(s+\hbar \varkappa / \pi)} .
\end{aligned}
$$

Для асимптотики этих коэффициентов при больших $s$ из (4.17) сразу следует, что они убывают как $O(1 / s)$, отличаясь при этом только знаком:

$$
\begin{aligned}
C_{s} & \rightarrow A \frac{\sin [\hbar(\varkappa+i \omega) / 2] \sin [\hbar(\varkappa-i \omega) / 2]}{\pi s \sin \hbar \varkappa}, s \rightarrow \infty, \\
\bar{C}_{s} & \rightarrow A \frac{\sin [\hbar(\bar{\varkappa}+i \omega) / 2] \sin [\hbar(\bar{\varkappa}-i \omega) / 2]}{\pi s \sin \hbar \varkappa}= \\
& =-A \frac{\sin [\hbar(\varkappa+i \omega) / 2] \sin [\hbar(\varkappa-i \omega) / 2]}{\pi s \sin \hbar \varkappa}, \quad s \rightarrow \infty .
\end{aligned}
$$

Тем самым сумма $C_{s}+\bar{C}_{s}$ убывает как $O\left(1 / s^{2}\right)$, что гарантирует как сходимость ряда (4.8) для $\psi_{\text {out }}(a)$, так и мероморфность функции $f(z)$ на всей комплексной плоскости. Из (4.18) также следует сходимость ряда

$$
\psi_{\text {out }}(a+i y)=\sum_{s}\left(C_{s} e^{-i \varkappa_{s} y}+\bar{C}_{s} e^{-i \bar{\varkappa}_{s} y}\right)
$$


при $y \in(-\hbar,+\hbar)$. Однако, если в ряде $(4.8)$ для $\psi_{\text {out }}(a)$ логарифмические расходимости от $\sum_{s} C_{s}$ и $\sum_{s} \bar{C}_{s}$ сокращаются за счет противоположных знаков в асимптотиках (4.18), то в $\psi_{\text {out }}(a \pm i \hbar)$ эти слагаемые входят с неравными при $\varkappa \neq 0, \pi / \hbar$ постоянными множителями $e^{ \pm i \hbar \varkappa}$ :

$$
\psi_{\text {out }}(a \pm i \hbar)=e^{\mp i \hbar \varkappa} \sum_{s} C_{s}+e^{ \pm i \hbar \varkappa} \sum_{s} \bar{C}_{s}
$$

Поэтому в данной задаче как в теории возмущений по $\hbar$, так и в рассматриваемом приближении “широкой ямы" функция $\psi(z)$ имеет логарифмические особые точки при $z=$ $\pm a+i n \hbar, n \neq 0$, и соответствующие вертикальные разрезы.

Заметим также, что сравнение (4.9) и (4.14) позволяет установить следуюшее тождество:

$$
\begin{gathered}
\sum_{s=0}^{\infty}\left[\frac{\Gamma[s+(\varkappa+i \omega) / 2] \Gamma[s+(\varkappa-i \omega) / 2]}{\Gamma(s+1) \Gamma(s+\varkappa)}-\right. \\
\left.\quad-\frac{\Gamma[s+1-(\varkappa-i \omega) / 2] \Gamma[s+1-(\varkappa+i \omega) / 2]}{\Gamma(s+1) \Gamma(s+2-\varkappa)}\right]= \\
=\frac{\pi \sin \pi \varkappa}{\sin [\pi(\varkappa+i \omega) / 2] \sin [\pi(\varkappa-i \omega) / 2]}
\end{gathered}
$$

которое справедливо для всех $\varkappa$ из интервалов $0 \leqslant \varkappa \leqslant 1$ и $\varkappa=1+i \alpha$ и всех действительных $\omega$.

Коэффициенты внутреннего решения $A_{n}$ находятся с помошью соотношений $(2.20)$ непосредственно по значениям функции $f(z)$ в точках $z= \pm i \omega_{n}$. Следует только выбирать то из выражений $(2.20)$, которое обеспечивает убывание $A_{n}$ с ростом $|n|$. Учитывая последнее обстоятельство, для $n \geqslant 0$ получим

$$
\begin{aligned}
A_{n} & =\frac{\hbar V_{0}}{i \operatorname{sh} \hbar \omega} e^{-i \omega_{n} a} f\left(-i \omega_{n}\right)= \\
& =A^{\prime} \frac{\hbar V_{0} e^{-i \omega_{n} a}}{i \operatorname{sh} \hbar \omega} \frac{\Gamma[n+\hbar(\varkappa+i \omega) / 2 \pi] \Gamma[n+1-\hbar(\varkappa-i \omega) / 2 \pi]}{\Gamma(n+1) \Gamma(n+1+i \hbar \omega / \pi)} .
\end{aligned}
$$

Коэффициенты $A_{n}, n<0$, определяются из (4.22) как $A_{-n}^{*}$. Заметим, что выражения (4.22) для коэффициентов $A_{n}$ можно также получить, решая непосредственно систему уравнений $(2.14),(2.14 \mathrm{a})$ внутренней задачи в том же приближении, когда опускаются все слагаемые, содержащие убываюшие экспоненты $e^{-2 \pi n a / \hbar}, n>0$. Спектральное уравнение (4.15) при этом есть не что иное, как условие действительности коэффициента $A_{0}$. Далее из (4.22) для асимптотики $A_{n}$ при $|n| \rightarrow \infty$ находим

$$
A_{n} \rightarrow \pm A^{\prime} \frac{\hbar V_{0} e^{\mp i \omega a}}{i|n| \operatorname{sh} \hbar \omega} e^{-2 \pi|n| a / \hbar}, \quad n \rightarrow \pm \infty
$$

Это с точностью до множителя совпадает с первым порядком теории возмущений (3.7), что полностью подтверждает все выводы относительно поведения $\psi(z)$ в рамках приближения "широкой ямы", полученные выше на основе внешнего решения. 
Рассмотрим теперь область применимости приближения (4.6), критерием которой является малость невязки, возникающей при переходе от уравнения (4.4) к (4.6) для $n>0$. Эта невязка есть $e^{-i \omega_{n} a} f\left(-i \omega_{n}\right)$, где

$$
f\left(-i \omega_{n}\right)=\frac{\Gamma[n+\hbar(\varkappa+i \omega) / 2 \pi] \Gamma[n+1-\hbar(\varkappa-i \omega) / 2 \pi]}{\Gamma(n+1) \Gamma(n+1+i \hbar \omega / \pi)} .
$$

Очевидно, что если

$$
\frac{\hbar \varkappa}{\pi}<1, \quad \frac{\hbar \omega}{\pi}<1
$$

то поведение $\left|f\left(-i \omega_{n}\right)\right|$ при $n \geqslant 1$ является вполне монотонным с фактическим выходом на асимптотику $\left|f\left(-i \omega_{n}\right)\right| \rightarrow 1 / n$ уже при конечных $n \gg 1$. В этих условиях наличие убывающей экспоненты $e^{-2 \pi n a / \hbar}$ гарантирует малость невязки для всех $n \geqslant 1$, и тем самым приближение “широкой ямы” заведомо применимо до тех пор, пока глубина ямы удовлетворяет условию

$$
V_{0}<\frac{4}{\hbar^{2}}
$$

Более того, легко видеть, что если

$$
\hbar \omega \ll 1, \quad \hbar \varkappa \ll 1,
$$

так что уравнение (2.3) переходит в (3.2) и дает

$$
\hbar^{2} V_{0} \ll 1
$$

то уравнение (4.15) превращается в (3.5) для четных уровней соответствующей задачи Шредингера (1.5), а выражение (4.22) для коэффициентов $A_{n}$ в том же пределе переходит в соотношение (3.7), соответствуюшее первому порядку теории возмушений. Тем самым для "широкой ямы” уравнение Шредингера (1.5) может быть обосновано в качестве нулевого приближения для уравнения (1.1) при условиях (4.26), (4.26а) на параметры, более слабых, чем (3.1), (3.3), которые были необходимы для решения уравнения (1.1) с помощью теории возмушений по степеням $\hbar$ в окрестности шредингеровской волновой функции.

При этом если глубина ямы $V_{0}$ растет от нуля до критического значения $4 / \hbar^{2}$, то найденное в приближении (4.6) решение $\psi(x)$ уравнения (1.1) в целом повторяет поведение шредингеровской волновой функции, качественно отличаясь от последней только наличием переходных областей размером $O(\hbar / \pi)$ между внутренним (почти) косинусом и убываюшей (почти) экспонентой вне ямы и более сложной зависимостью от $V_{0}$ базовых волновых чисел внутреннего и внешнего решений $\omega\left(V_{0}\right)$ и $\varkappa\left(V_{0}\right)$ (и тем самым $\left.E\left(V_{0}\right)\right)$, которые вне рамок условия (4.26) определяются из значительно более сложных по сравнению с (3.2), (3.5) уравнений (2.3) и (4.15). Численный анализ этих уравнений тем не менее показывает, что при глубине ямы меньше критической для каждого уровня энергии $E\left(V_{0}\right)$ соответствуюшее базовое волновое число $\varkappa\left(V_{0}\right)$ внешнего решения растет с ростом $V_{0}$, поэтому растет и скорость убывания решения $\psi(x)$ вне ямы, как и у его шредингеровского аналога. 
Если же глубина ямы $V_{0}$ превышает критическое значение $4 / \hbar^{2}$, то для низколежащих уровней, для которых

$$
|E|>\frac{4}{\hbar^{2}},
$$

поведение $\psi(x)$ сушественно меняется за счет того, что теперь $\varkappa=\pi / \hbar+i \alpha$, где $\alpha$ определяется из (1.16). Для таких глубоких уровней, в полной аналогии со случаем $\delta$-образного потенциала, поведение $\psi(x)$ во внешней области становится осциллируюшим с периодом $2 \pi / \alpha$ и амплитудой, которая убывает как $e^{-\pi|x| / \hbar}$ независимо от положения уровня и глубины ямы, а при дальнейшем росте $V_{0}$ увеличивается только частота осцилляций. Сами же уровни энергии в области (4.27) находятся из уравнения

$$
e^{2 i \omega a}=\frac{\Gamma(-i \hbar \omega / \pi)}{\Gamma(i \hbar \omega / \pi)} \frac{\Gamma[1 / 2+i \hbar(\omega+\alpha) / 2 \pi] \Gamma[1 / 2+i \hbar(\omega-\alpha) / 2 \pi]}{\Gamma[1 / 2-i \hbar(\omega+\alpha) / 2 \pi] \Gamma[1 / 2-i \hbar(\omega-\alpha) / 2 \pi]} .
$$

Пока глубина ямы $V_{0}$ незначительно превышает критическое значение $4 / \hbar^{2}$, так что $\hbar \alpha / 2 \pi, \hbar \omega / 2 \pi$ ограничены сверху порядком $O(1)$, приближение (4.6) по-прежнему вполне оправдано, поскольку в этих условиях поведение последовательности $\left|f\left(-i \omega_{n}\right)\right|$ при $n \geqslant 1$ незначительно отличается от случая (4.25). Наличие убывающих экспонент $e^{-2 \pi n a / \hbar}$ снова гарантирует малость невязки для всех $n \geqslant 1$, а поправки, обусловленные вторым слагаемым $e^{-i \omega_{n} a} f\left(-i \omega_{n}\right)$ в уравнении $(4.4)$, эффективно учитываются с помощью теории возмущений по степеням $\lambda$. Положение уровней при этом с высокой точностью находятся из системы уравнений (2.3) и (4.28). Сильная нелинейность последней допускает только численный анализ, результаты которого можно наглядно описать при условии, что $4 / \hbar^{2} \gg 1$. Заметим, что в рамках исходной задачи последнее условие является вполне реалистическим. Если оно выполняется, то нижние уровни в соответствуюшем уравнении Шредингера (1.5) практически совпадают с уровнями в бесконечно глубокой яме шириной $2 a$, которые для четного случая находятся из уравнения

$$
e^{2 i \omega a}=-1 .
$$

В то же время из соотношений (2.3) и (4.28) следует, что при этих условиях нижние уровни в уравнении (1.1) оказываются сдвинутыми вверх по отношению к шредингеровским, т.е. для этих уровней в уравнении (1.1) эффективная ширина ямы становится меньше, чем геометрическая.

С дальнейшим ростом глубины ямы этот эффект становится все более выраженным и может быть получен в аналитической форме непосредственно из уравнения (4.28) для $V_{0}$, значительно превьшающих критическое значение

$$
V_{0} \gg \frac{4}{\hbar^{2}}
$$

т.е. когда для нижних уровней с $\omega \ll \alpha$ начинает выполняться условие

$$
\frac{\hbar \alpha}{2 \pi} \gg 1
$$

Для этого случая в нулевом приближении по $\omega / \alpha$ уравнение $(4.28)$ может быть представлено в виде, аналогичном (4.29), именно

$$
e^{2 i \omega a_{\mathrm{eff}}}=-1,
$$


причем эффективная ширина ямы $a_{\mathrm{eff}}=a-\Delta a$ оказывается меньше геометрической на величину

$$
\Delta a=\frac{\hbar}{\pi} \ln \left(\frac{\hbar \alpha}{2 \pi}\right)
$$

Из соотношений (4.32), (4.33) следует, что в области (4.30), (4.31) с ростом $V_{0}$ нижние уровни постоянно сдвигаются вверх относительно дна ямы, хотя и весьма медленно дваждылогарифмически, поскольку для таких $V_{0}$ при $\omega \ll \alpha$ будем иметь

$$
\hbar \alpha=\ln \left(\hbar^{2} V_{0}\right) .
$$

Заметим также, что такие "нижние уровни" теперь заполняют достаточно большой интервал энергии, так как условия $\omega \ll \alpha$ и (4.31) теперь могут быть совместны с $\hbar \omega \gg 1$.

На качественном уровне этот эффект роста значений энергии нижних уровней с увеличением $V_{0}$ есть прямое следствие специфической конечно-разностной структуры кинетического члена (1.3) в энергетическом функционале (1.1a).Действительно, основное состояние является минимумом функционала (1.1a). Поэтому с увеличением глубины волновая функция стремится сконцентрироваться внутри ямы. Однако, в отличие от дифференциального уравнения Шредингера (1.5), для которого это достигается за счет двух факторов - во-первых, за счет уменьшения коэффициента при убывающей экспоненте и, во-вторых, за счет увеличения скорости убывания этой экспоненты, для уравнения (1.1) второй фактор ограничен из-за структуры функционала кинетической энергии $(1.3),(1.4)$. Именно, волновая функция $\psi(x)$, убывающая вне ямы быстрее, чем $e^{-\pi|x| / \hbar}$, с неизбежностью будет иметь фурье-образ $\widetilde{\psi}(k)$, убывающий медленнее, чем $e^{-\hbar|k| / 2}$ при $|k| \rightarrow \infty$, и тем самым бесконечную кинетическую энергию. Следовательно, с ростом $V_{0}$ мы сначала, в докритической области (4.25a), будем получать повторение нормальной шредингеровской картины (уменьшение как коэффициента, так и глубины проникновения за пределы ямы), а затем, когда $V_{0}$ достигнет критического значения, глубина проникновения в запрешенную область станет порядка $O(\hbar / \pi)$ и более уменьшаться не будет, а при дальнейшем росте $V_{0}$ убывать будет только коэффициент при экспоненте. В результате в случае уравнения (1.1) положительньй вклад в энергию уровня за счет кинетической энергии убывает с ростом глубины ямы в области (4.30) значительно медленнее, чем для уравнения (1.5), что и приводит к наблюдаемому эффекту сдвига нижних уровней вверх по отношению к шредингеровским.

Следует также отметить, что наряду с ростом уровней в области (4.30), (4.31) происходят качественные изменения и в структуре самой волновой функции $\psi(x)$. Это связано с тем, что при условии (4.31) коэффициенты $A_{n}$ и $C_{s}, \bar{C}_{s}$ сначала растут как $(\hbar \alpha / 2 \pi)^{|n|}$ и $(\hbar \alpha / 2 \pi)^{s}$, соответственно, а потом, при достаточно больших $|n|$ и $s$, выходят на убывающую асимптотику. В результате происходит расширение переходной области между внутренним косинусом и внешней экспонентой на величину порядка $(\hbar / 2 \pi) \ln (\hbar \alpha / 2 \pi)$, что и приводит к эффективному сужению ямы (4.33).

Такой эффект сужения ямы и роста уровней, однако, имеет место только в рамках приближения (4.6), поскольку является прямым следствием структуры спектрального уравнения (4.28), которое выводится из этого приближения. Между тем в области (4.30) переход от уравнения (4.4) к (4.6) уже неочевиден и требует специального изучения, так как в этом случае $|\hbar(\varkappa \pm i \omega) / 2 \pi| \gg 1$, что сушественно влияет на величину $\left|f\left(-i \omega_{n}\right)\right|$ 
и тем самым на величины невязки при конечных $n \geqslant 1$. В наиболее последовательном подходе применимость (4.6) как начального приближения для уравнения (4.4) следует обеспечивать малостью поправок, возникающих при учете второго слагаемого в (4.4) в рамках теории возмушений по степеням $\lambda$. Такая теория возмущений, однако, обладает своей спецификой и поэтому будет детально рассмотрена в отдельной работе. Здесь же мы приведем менее строгий, но достаточно короткий способ оценки пределов применимости приближения (4.6), приводяший тем не менее к правильным конечным результатам. Его суть состоит в том, чтобы обеспечить для слагаемого с растущей экспонентой в уравнении (4.4) статус главного, для чего потребуем вьполнения следующего условия:

$$
e^{-2 \pi n a / \hbar}\left|f\left(-i \omega_{n}\right)\right| \ll e^{2 \pi n a / \hbar}\left|f\left(i \omega_{n}\right)\right|
$$

на вешественной положительной полуоси по теперь уже непрерьвной переменной $n$ вне малых окрестностей целых точек, где $f\left(i \omega_{n}\right)=0$. Чтобы результат такой оценки был максимально нагляден, потребуем выполнения еше более сильного условия, чем (4.35), а именно

$$
e^{-2 \pi n a / \hbar}\left|f\left(-i \omega_{n}\right) \Gamma(n+1)\right| \ll e^{2 \pi n a / \hbar}\left|f\left(i \omega_{n}\right) \Gamma(1-n)\right| .
$$

Множители $\Gamma(n+1)$ и $\Gamma(1-n)$ при этом значительно увеличивают степень выполнения исходного неравенства (4.35) при $n \gg 1$. Поскольку для глубоких уровней, для которых выполняется условие (4.27), в области (4.30) будем иметь $|\hbar(\omega \pm \alpha) / 2 \pi| \gg 1$, далее из (4.35a) с помошью соответствуюшей асимптотики для $|\Gamma(x+i y)|$ при $|y| \rightarrow \infty$ находим

$$
\frac{\left|(\hbar \alpha / 2 \pi)^{2}-(\hbar \omega / 2 \pi)^{2}\right|}{\hbar \omega / \pi} \ll e^{2 \pi a / \hbar} .
$$

Заметим, что при этом как в левой, так и в правой частях неравенства (4.35а) возникают одинаковые множители $\exp [-\hbar(|\alpha+\omega|+|\alpha-\omega|) / 4+\hbar \omega / 2]$, которые в окончательный ответ не входят, но экспоненциально малы при $\alpha \gg \omega$. Таким образом, оценка только $\left|f\left(-i \omega_{n}\right)\right|$ при $\alpha \gg \omega$ привела бы к экспоненциально малой при $\hbar \alpha \gg 1$ величине, но для фактической малости невязки в уравнении (4.4) этого недостаточно, так как при этом аналогичный множитель $e^{-\hbar \alpha / 2}$ возникает и в $\left|f\left(i \omega_{n}\right)\right|$. Поэтому неравенство (4.35а) обеспечивает в данном случае более строгую оценку малости невязки в уравнении (4.4).

При этом для самых нижних уровней, для которых $\hbar \omega$ порядка $O(1)$, из $(4.36)$ следует, что

$$
\frac{\hbar}{\pi} \ln \left(\frac{\hbar \alpha}{2 \pi}\right) \ll a
$$

Таким образом, предписываемое уравнениями (4.32), (4.33) сужение ямы и рост нижних уровней могут быть строго обоснованы только пока эти эффекты достаточно малы. Тем не менее эти эффекты вполне наблюдаемы, так как в силу дваждылогарифмического характера они имеют место в весьма широком диапазоне изменения глубины ямы $V_{0}$, именно

$$
4 \ll \hbar^{2} V_{0} \ll \exp \left[2 \pi e^{\pi a / \hbar}\right],
$$

который очень быстро (дваждыэкспоненциально) увеличивается с ростом ширины ямы. Так, при $a \simeq 2 \hbar$ в неравенстве (4.38) ограничение на $\hbar^{2} V_{0}$ сверху задается числом порядка $1000^{1000}$. 
Численные расчеты, основанные на решении непосредственно исходных систем (2.14), (2.19), подтверждают такое поведение решения уравнения (1.1) в области больших глубин. В частности, при $\hbar=1, a=\pi$ и $\alpha=2000$, что удовлетворяет неравенству (4.37), поправки к решению, основанному на приближении (4.6), имеют порядок $10^{-7}$, что показывает высокую точность приближения “широкой ямы” в области его применимости. Более того, численный расчет для значений $V_{0}$, выходящих за рамки неравенства (4.38), показывает, что рост уровней по-прежнему имеет место. Так, при $\hbar=1, a=0.7 \pi$ и $\alpha=2000$ (при этом $\alpha$ уже одного порядка с $2 \pi e^{\pi a} \simeq 7000$ ) поправки к приближению (4.6) уже имеют порядок $10^{-3}$, а нижний уровень имеет базовое волновое число $\omega=4.75$, что почти в семь раз превышает соответствуюшее значение $\omega=0.7$ для основного состояния в уравнении Шредингера (1.5).

Получить аналитические результаты для значений глубины ямы $V_{0}$, выходящих за рамки неравенства (4.38), не удается. Из (4.36) следует лишь, что для неограниченно больших $V_{0}$ приближение (4.6) сохраняет свой статус нулевого приближения для уравнения (1.1), если $\omega \simeq \alpha$, т.е. для уровней с энергиями порядка $V_{0} / 2$ относительно дна ямы. Однако нет никаких оснований считать, что при этом в задаче не может быть более низколежащих уровней. K сожалению, непосредственный численный счет при очень больших $\alpha$ также сильно затруднен крайне медленной сходимостью соответствуюших рядов и произведений - остаточный член оказывается пропорционален $\alpha^{2} / N$, так что для данного $\alpha$ сходимость достигается лишь при $N \gg \alpha^{2}$. Поэтому про поведение нижних уровней за границей применимости приближения (4.6) можно определенно утверждать только то, что они заведомо не убывают.

\section{5. ЗАКЛЮЧЕНИЕ}

В настояшей работе ставилась задача показать на примере уравнения (1.1), что уравнения такого типа с конечными чисто мнимыми сдвигами (радиального) аргумента на $\pm i \hbar$, естественным образом возникающие в ряде актуальных проблем КТП [3]-[5], [6], по многим весьма принципиальным аспектам радикально отличаются от своих дифференциальных аналогов. В частности, в случае уравнения (1.1) мы сталкиваемся с ситуацией, когда структура спектра и свойства волновых функций сушественным образом зависят от соотношения между $\hbar$ и параметрами потенциала $V(x)$, причем вполне возможно, что при $\hbar \ll 1$ решение уравнения (1.1) не будет иметь ничего обшего с решением его дифференциального аналога - нерелятивистского уравнения Шредингера (1.5). В частности, для потенциала в виде прямоугольной потенциальной ямы (1.21) эта ситуация реализуется при выполнении условий (4.27) и (4.30).

Следует также заметить, что рассмотренный в разделе 4 метод квазиточного решения уравнения (1.1) для прямоугольной ямы может быть обобшен таким образом, что для любых значений параметров задачи он будет приводить к формально точному аналитическому ответу. Фактически это обобщение сводится к суммированию ряда теории возмушений по степеням $\lambda$ с приближением (4.6) в качестве нулевого члена в некоторое условное компактное выражение, которое позволяет доказать сходимость ряда к точному решению для всех $\lambda<1$. Однако детальный анализ этого выражения вне области применимости приближения (4.6) весьма сложен, так как при этом необходимо удерживать слишком большое число членов ряда, каждым из которых пренебречь невозможно. 
Так что утверждать что-либо определенное о поведении уровней и волновых функций для произвольных значений параметров задачи пока не представляется возможным.

С другой стороны, приближение (4.6) с учетом нескольких первых поправок по $\lambda$ позволяет получить практически точное решение уравнения (1.1) в весьма широком диапазоне значений параметров задачи, актуальных с точки зрения физических приложений. Фактически единственный принципиальный нерешенный вопрос, который заведомо оказывается вне рамок этого метода, - это поведение спектра и волновых функций при $V_{0} \rightarrow \infty$ для фиксированных $\hbar$ и $a$. Однако физическая актуальность этого вопроса проблематична, так как уже при $a \simeq 2 \hbar$ приближение (4.6) применимо вплоть до $\hbar^{2} V_{0} \simeq 1000^{1000}$, что во многих аспектах может рассматриваться как бесконечно большое число.

Благодарности. Данная работа выполнена при финансовой поддержке РФФИ (грант № 00-15-96577).

\section{Список литературы}

[1] A. A. Logunov, A. N. Tavkhelidze. Nuovo Cimento. 1963. V. 29. P. 380.

[2] В. Г. Кадышевский. ЖЭТФ. 1963. Т. 46. С. $654 ;$ С. 872 ; ДАН СССР. 1965. Т. 160. C. 573.

[3] V. G. Kadyshevsky. Nucl. Phys. B. 1968. V. 6. P. 125; V. G. Kadyshevsky, M. D. Mateev. Nuovo Cimento A. 1968. V. 55. P. 275.

[4] V. G. Kadyshevsky, R. M. Mir-Kasimov, N. B. Skachkov. Nuovo Cimento A. 1968. V. 55. Р. 233; В. Г. Кадышевский, Р. М. Мир-Касимов, М. Фриман. ЯФ. 1969. Т. 9. C. 646; M. Freeman, M. D. Mateev, R. M. Mir-Kasimov. Nucl. Phys. B. 1969. V. 12. Р. 197; В. Г. Кадьиевский, М. Д. Матеев, Р. М. Мир-Касимов. ЯФ. 1970. Т. 11. С. 692 ; В. Г. Кадышевский, Р. М. Мир-Касимов, Н. Б. Скачков. ЭЧАЯ. 1972. Т. 2. С. 635; Н. Б. Скачков, И. Л. Соловцов. ЭЧАЯ. 1978. Т. 9. С. 5; А. Д. Донков, В. Г. Кадышевский, М. Д. Матеев. ТМФ. 1982. Т. 50. С. 360.

[5] V. I. Savrin, V. B. Skachkov. Lett. Nuovo Cimento. 1980. V. 29. P. 363; V. I. Savrin, A. V. Sidorov, V. B. Skachkov. Hadronic J. 1981. V. 4. P. 1642; B. A. Mamsees, В. И. Саврин, А. Н. Сисакян, А. Н. Тавхелидзе. ТМФ. 2002. Т. 132. № 2. С. 267.

[6] K. A. Sveshnikov. Phys. Lett. A. 1989. V. 136. Р. 1; K. А. Свешников. ТМФ. 1990. Т. 82. C. 55 .

[7] А. О. Гельфонд. Исчисление конечных разностей. М.: Физматгиз, 1967.

[8] К. А. Свешников, П. К. Силаев. ТМФ. 1996. Т. 108. С. 212; K. Sveshnikov, P. Silaev, I. Cherednikov. Mod. Phys. Lett. A. 1997. V. 12. P. 465.

[9] Л. В. Канторович, В. И. Крылов. Приближенные методы высшего анализа. М.: ГИТТЛ, 1949.

[10] В. В. Воеводин. Численные методы алгебры. Теория и алгоритмы. М.: Наука, 1966; B. B. Воеводин, E. E. Тыртышников. Вычислительные процессы с теплицевыми матрицами. М.: Наука, 1987.

Поступила в редакцию 31.III.2002 г.

4 Теоретическая и математическая физика, т. 132, № 3, 2002 г. 